\title{
Turnover and Function of Noncoding RNA Polymerase II Transcripts
}

\author{
M.J. Dye, ${ }^{*}$ N. Gromak,* D. Haussecker ${ }^{\dagger}$ S. West, ${ }^{*}$ and N.J. Proudfoot* \\ *Sir William Dunn School of Pathology, University of Oxford, Oxford, OX1 3RE, United Kingdom; \\ ${ }^{\dagger}$ Stanford School of Medicine, Department of Pediatrics, Stanford, California 94305
}

\begin{abstract}
In the past few years, especially since the discovery of RNA interference (RNAi), our understanding of the role of RNA in gene expression has undergone a significant transformation. This change has been brought about by growing evidence that RNA is more complex and transcription more promiscuous than has previously been thought. Many of the new transcripts are of so-called noncoding RNA (ncRNA); i.e., RNA that does not code for proteins such as mRNA, or intrinsic parts of the cellular machinery such as the highly structured RNA components of ribosomes (rRNA) and the small nuclear RNA (snRNA) components of the splicing machinery. It is becoming increasingly apparent that ncRNAs have very important roles in gene expression. This paper focuses on work from our laboratory in which we have investigated the roles and turnover of ncRNA located within the gene pre-mRNA, which we refer to as intragenic ncRNA. Also discussed are some investigations of intergenic ncRNA transcription and how these two classes of ncRNA may interrelate.
\end{abstract}

In eukaryotes, protein encoding genes are transcribed by RNA polymerase II (pol II). Typically, pol II transcription initiates within the promoter region of the gene that is encoded in the DNA template, and terminates at some point downstream from the poly(A)-addition site in the $3^{\prime}$-flanking region (see the diagram of the human $\beta$-globin gene; Fig. 1A). pol II transcription initiating at the promoter generates a pre-mRNA copy of this DNA template that is conventionally subdivided into the following regions: the $5^{\prime}$-untranslated region $\left(5^{\prime} \mathrm{UTR}\right)$; the protein-encoding section of the pre-mRNA composed of exons interspersed with noncoding intron sequences; and the 3'UTR including the poly(A) signal. The pre-mRNA also extends beyond the poly(A)-site sequences into the 3 -flanking region up to the point of transcription termination (Fig. 1B). As $3^{\prime}$-flanking regions are highly unstable, few have been accurately defined. However, for the mouse $\beta$-globin (Tantravahi et al. 1993), the human $\beta$ and $\varepsilon$-globin (Dye and Proudfoot 2001), and the mouse serum albumin (West et al. 2006a) genes, it appears that the $3^{\prime}$ terminus of the pre-mRNA lies between 1 and $2 \mathrm{~kb}$ downstream from the poly(A) site. Although the full extent of the $3^{\prime}$-flanking region of the majority of premRNAs is unknown, it is well established that most premRNAs conform to the arrangement of coding and noncoding elements seen in the $\beta$-globin gene. Different gene pre-mRNAs show great variation in terms of length and complexity. Many contain more exons and longer introns that may be spread over hundreds of kilobases of the genome (Strachan and Read 1999). Therefore, different gene pre-mRNAs may diverge greatly in the ratio of coding/noncoding sequence.

It is immediately apparent from Figure 1B that even the relatively small $\beta$-globin gene pre-mRNA is predominantly composed of ncRNA. This high ratio of ncRNA to coding RNA is typical of most pre-mRNAs. Indeed, on some gene maps, such as that of the dystrophin gene, exons appear as tiny specks in a vast ocean of ncRNA. Some of this ncRNA is very important. The $5^{\prime}$ UTR and $3^{\prime} \mathrm{UTR}$, intronic splice signals, and poly(A)-site sequences guide essential steps in gene expression. For example, processing of the $\beta$-globin pre-mRNA by splicing out of introns and endonucleolytic cleavage followed by polyadenylation at the poly(A)-addition site yields the mature mRNA, shown in Figure 1C. Furthermore, the $5^{\prime}$ UTR and $3^{\prime} \mathrm{UTR}$ are retained in the mRNA where they have important roles in nuclear export, mRNA stability, and cytoplasmic localization (Moore 2005). 3'UTRs are also involved in the control of mRNA translation which, in an estimated $30 \%$ of genes, involves interaction with another class of ncRNA, termed microRNAs (miRNAs) (Lewis et al. 2005). However, the majority of ncRNAs derived from gene introns and $3^{\prime}$ flanking regions are of unknown function. Much of this ncRNA has been assumed to be simply "junk" transcript, which has arisen merely as a consequence of gene evolution and as such is rapidly discarded by RNA degradation. The large energetic cost of this dominant gene transcript is apparently tolerated by mammalian nuclei. This view of ncRNA as predominantly nonfunctional transcript is now challenged by the identification of growing numbers of small regulatory RNAs, namely, small nucleolar RNAs (snoRNAs) and miRNAs (Bartel 2004; Mattick and Makunin 2006). These findings substantially alter the view that ncRNA is functionless.

In recent years, it has become apparent that for many genes, pre-mRNA processing occurs cotranscriptionally. This means that addition of the cap structure to the $5^{\prime}$ end of the pre-mRNA, excision of introns, endonucleolytic cleavage at the poly(A) site, and even addition of the poly(A) tail occur as pol II produces the pre-mRNA copy of the DNA template (Maniatis and Tasic 2002; Proudfoot et al. 2002). Indeed, various lines of evidence indicate that pre-mRNA processing and pol II transcription are coupled 
A

$\beta$-globin gene

B $\beta$-globin pre-mRNA



3'UTR 3' FLANKING REGION

C

$\beta$-globin mRNA

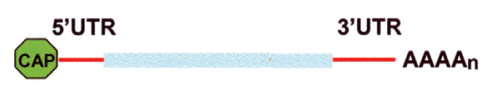

Figure 1. Structure of the human $\beta$-globin gene. $(A)$ Diagram of the human $\beta$-globin gene. The promoter and terminator sequences are represented by the green and red boxes, respectively. The arrow indicates the transcription start site. $(B)$ Diagram of the human $\beta$-globin pre-mRNA. Unlabeled components of the pre-mRNA are listed in the key below the diagram. $(C)$ Diagram of the human $\beta$-globin mRNA.

events; in many different systems, we see that perturbation of one process may effect a change in the other. One of the earliest demonstrations of the cotranscriptional nature of pre-mRNA processing was the finding that mutation of the $\alpha$-globin gene poly(A) site perturbed transcription termination in the $\alpha$-globin gene $3^{\prime}$-flanking region (Whitelaw and Proudfoot 1986). Evidence for the coupling of transcription and pre-mRNA processing led to the generation of models showing physical linkage of components of the transcription and pre-mRNA processing apparatuses (Proudfoot et al. 2002). The transcription-RNA processing complex finds its most ambitious expression in the notion that large transcription "factories" exist as conglomerates of polymerase and processing factors at specific nuclear locations (Bartlett et al. 2006). From a purely practical viewpoint, the coupling of transcription and premRNA processing provides a convenient "handle" for the experimentalist to analyze these complex processes and their potential interactions.

As mentioned above, specific roles in pre-mRNA processing and mRNA stability have been assigned to discrete intragenic ncRNA sequences. However, there is little understanding of the roles and turnover of the majority of intragenic ncRNA located in the pre-mRNA $3^{\prime}$-flanking region and introns. This is partly due to the difficulty of examining such rapidly metabolized RNA species. In our laboratory, we have refined and developed techniques that have enabled us to overcome some of the problems inherent in studying unstable nascent RNA. This has enabled us to make several interesting observations concerning the biology of the $3^{\prime}$-flanking region and intron ncRNA transcripts discussed below.

\section{ROLES AND TURNOVER OF 3'-FLANKING REGION NONCODING RNA TRANSCRIPTS}

For several years, we have mapped nascent pre-mRNAs in the 3'-flanking regions of genes with the goal of finding sites of transcriptional termination. In particular, we have employed the nuclear run-on (NRO) technique, in which RNA molecules are radioactively end-labeled as they are transcribed by endogenous RNA polymerase in vivo. NRO analysis yields two types of data: It shows the distribution of active RNA polymerase on the DNA template of a given gene and the extent of the gene pre-mRNA. NRO analysis of 3'-flanking region transcripts of the human $\beta$ globin gene gives the pattern of hybridization signals shown in Figure 2A. Strong hybridization signals over probes 4-10 that span between 0 and $1.6 \mathrm{~kb}$ downstream from the poly(A) site are followed by background hybridization signals over probes $\mathrm{A}, \mathrm{B}$, and $\mathrm{C}$, which lie immediately downstream from probe 10 . These data show the presence of active pol II throughout the $\beta$-globin 3'flanking region, extending up to $1.6 \mathrm{~kb}$ downstream from the poly(A) site. The background level hybridization signals beyond probe 10 , over probes $\mathrm{A}, \mathrm{B}$, and $\mathrm{C}$, indicate that pol II transcriptional termination takes place soon after this part of the $3^{\prime}$-flanking region has been transcribed (Dye and Proudfoot 1999). The role of specific 3'flanking region sequences in pol II transcription 
A

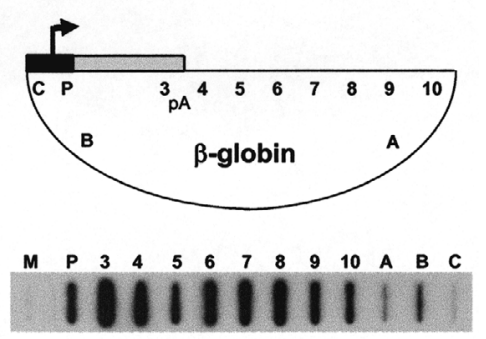

B
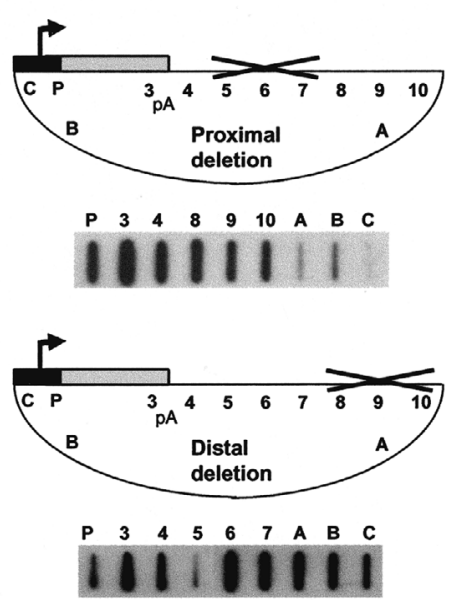

C
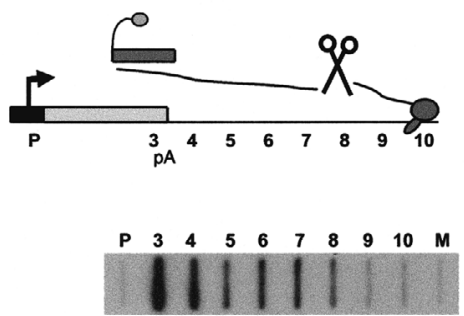

Figure 2. Nuclear Run On (NRO) analysis of $\beta$-globin gene transcripts. (A) NRO analysis of HeLa cells transiently transfected with the human $\beta$-globin gene construct shown in the diagram above the data panel. Characters in boldfaced type indicate the position of NRO probes. Probe M (M13 DNA) controls for background hybridization. pA indicates the position of the poly $(\mathrm{A})$ site. (B) NRO analysis of HeLa cells transiently transfected with derivatives of the human $\beta$-globin gene construct shown in the diagrams above the data panels. $(C)$ Hybrid selection NRO analysis of $\beta$-globin 3'-flanking region transcripts. (Single wavy line) pre-mRNA; (filled ovals) elongating pol II; (box with tail) position of the biotinylated selection probe; (scissors) CoTC activity.

termination was determined by deletion analysis. Although it was found that removal of the $800 \mathrm{bp}$ of $3^{\prime}$ flanking sequence lying proximal to the $\beta$-globin poly(A) site, corresponding to probes 5,6 , and 7 , has no effect on transcription termination, deletion of $3^{\prime}$-flanking region sequences lying more distal to the poly $(\mathrm{A})$ site, corresponding to probes 8,9 , and 10 , leads to a marked increase in readthrough transcription (Fig. 2B). This result shows that 3 -flanking region sequences, located between 1 and $1.6 \mathrm{~kb}$ downstream from the $\beta$-globin $\operatorname{poly}(\mathrm{A})$ site, are involved in transcriptional termination (Dye and Proudfoot 2001).

We next analyzed how these 3 -flanking region sequences mediate pol II termination. An early indication of the involvement of the $3^{\prime}$-flanking region transcript in the termination process was derived from the experiment shown in Figure 2C. In this experiment, which employs a hybrid selection technique to measure the continuity of nascent RNA molecules, we found that $\beta$-globin premRNA transcripts are not continuous throughout the 3 '-flanking region (Dye and Proudfoot 1999). Detailed analysis showed that transcript discontinuity occurs at several sites in a region lying between 1 and $1.6 \mathrm{~kb}$ downstream from the $\beta$-globin $\operatorname{poly}(\mathrm{A})$ site. Analysis of the $\varepsilon$-globin gene showed a similar but weaker effect (Dye and Proudfoot 2001). These data have far-reaching implications: First, they provide strong evidence that the ncRNA generated in the $\beta$-globin $3^{\prime}$-flanking region transcript is targeted by an RNA cleavage activity; second, the correspondence of the site of this cotranscriptional processing activity with the termination element indicates that cotranscriptional processing of the 3'-flanking region RNA might have an important role in transcription termination; and finally, the detection of noncontinuous nascent transcripts indicates that the linear pre-mRNA, depicted in Figure 1B as extending from the start site of transcription to the termination region, does not exist in vivo. The simplest way to interpret this last point is that although the $3^{\prime}$-flanking region pre-mRNA does not exist in vivo as a physically continuous molecule, it does, however, exist as a continuous sequence of information, some of which undergoes cotranscriptional processing.

Cotranscriptional cleavage (CoTC) of nascent transcripts generates two highly unstable products, termed the $5^{\prime}$ product and the $3^{\prime}$ product (Fig. 3A). Detailed analysis of the $5^{\prime}$ product was advanced by the development, in our laboratory, of an assay termed hscRACE (hybrid selection circular rapid amplification of cDNA ends). Here, the $5^{\prime}$ products of CoTC were circularized using T4 RNA ligase, then reverse-transcribed and polymerase chain reaction (PCR)-amplified using specific primers in the $3^{\prime}$-flanking region (West et al. 2006b). DNA sequencing of the hscRACE PCR products gives the surprising result that some of the $5^{\prime}$ products of CoTC are oligoadenylated. Furthermore, when components of the exosome complex are depleted from the transfected cells, $5^{\prime}$ products of CoTC are stabilized with a concomitant increase in the length of oligoadenylate tails (West et al. 2006b). These data show that oligoadenylation of mammalian ncRNAs can serve as a marker for degradation by the exosome, as has already been described for certain ncRNAs in yeast (Fig. 3C) (Wyers et al. 2005). It will be of interest to see the extent of oligoadenylation as a general mechanism in the turnover of ncRNAs.

Whereas analysis of the $5^{\prime}$ product of CoTC has yielded important insights into the turnover of ncRNA, investigation of the fate of the $3^{\prime}$ product revealed a connection between ncRNA turnover and pol II transcription termination (West et al. 2004). In this study, it was shown that the $3^{\prime}$ product of $\mathrm{CoTC}$ in the $\beta$-globin flanking region pre- 
A

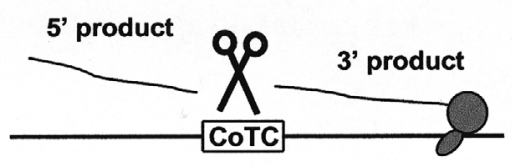

B
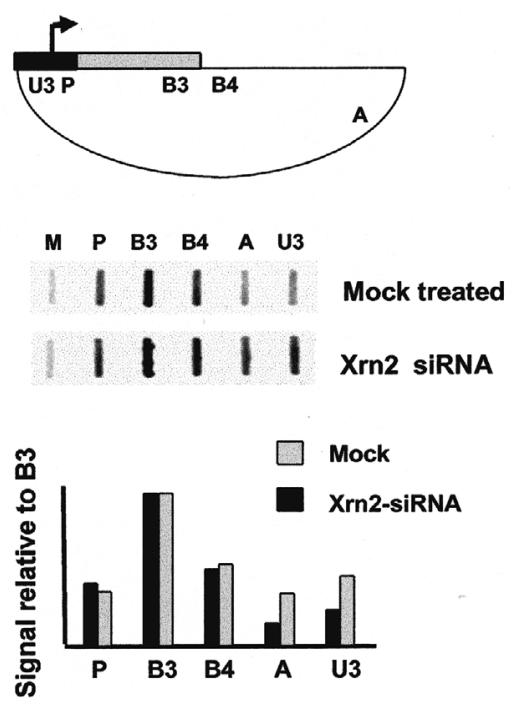

C

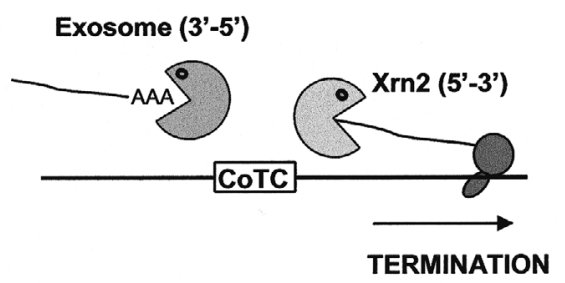

D

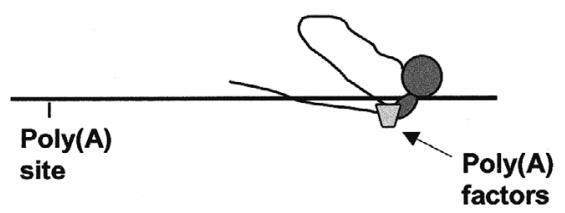

Figure 3. Turnover and roles of ncRNA in the $\beta$-globin $3^{\prime}$-flanking region. (A) Diagram of the products of CoTC in the 3'-flanking region. (Solid black line) DNA template; (single wavy line) pre-mRNA; (scissors) CoTC activity. $(B)$ Role of exonuclease Xrn2 in pol II transcription termination. (Upper panel) Diagram showing the $\beta$-globin gene construct; (middle data panel) NRO analysis of mock-treated or Xrn2 siRNAtreated HeLa cells transiently transfected with the $\beta$-globin gene construct; (bottom panel) graph showing corrected NRO hybridization signals in mock-treated or Xrn2 siRNA-treated cells. (C) Turnover of ncRNA in the $\beta$-globin $3^{\prime}$-flanking region following CoTC. Oligoadenylation of the $5^{\prime}$ product is indicated. The "Pac Man" symbols represent $3^{\prime}-5^{\prime}$ and $5^{\prime}-3^{\prime}$ exonucleases. $(D)$ Role of $3^{\prime}$-flanking region ncRNA in tethering poly(A)-site sequences to elongating pol II.
mRNA is a substrate of the nuclear $5^{\prime} \rightarrow 3^{\prime}$ exonuclease, Xrn2. Furthermore, it was demonstrated that transcriptional termination of the $\beta$-globin gene was diminished when Xrn2 was depleted by transfection of Xrn2-specific siRNAs, as shown in the NRO data in Figure 3B. The decrease in transcription termination efficiency is indicated by the increase in hybridization signals (due to transcriptional readthrough) over probes $\mathrm{A}$ and $\mathrm{U} 3$. This result was also confirmed by chromatin immunoprecipitation (ChIP) measuring pol II occupancy over the $\beta$-globin gene following Xrn2 depletion (West et al. 2004). At the same time, ChIP analysis in yeast showed that the homolog of $\mathrm{Xrn} 2$ in Saccharomyces cerevisiae, the $5^{\prime} \rightarrow 3^{\prime}$ exonuclease Rat1protein, localizes to the $3^{\prime}$ end of genes and is required for pol II termination (Kim et al. 2004). In some yeast genes, transcriptional termination is entirely dependent on the poly(A) site (Birse et al. 1998). It is therefore envisaged that Rat1protein engages with the pre-mRNA at the free $5^{\prime}$ end generated by poly(A)-site cleavage. It is currently thought that once $5^{\prime} \rightarrow 3^{\prime}$ exonuclease engages with the transcript at the free $5^{\prime}$ end, generated in a CoTC region or at the poly(A) site depending on the organism and specific gene, it then degrades the transcript until it "catches up" with pol II and then participates in the subsequent transcription termination process (Fig. 3C). This "torpedo" model was posited some time ago when the dependence of transcription termination on the poly(A) site was first identified (Whitelaw and Proudfoot 1986; Connelly and Manley 1988). At present, there are few details of the mechanism by which $5^{\prime} \rightarrow 3^{\prime}$ exonuclease promotes transcription termination. However, the absolute requirement for a $5^{\prime}$-phosphate terminus, generated by cleavage at the poly(A) site or the CoTC element rather than a $5^{\prime}$-hydroxyl terminus generated by hammerhead ribozyme cleavage, is a clear indication that RNA turnover is involved in the termination process (West et al. 2004). To further our research in this area, we have recently developed antibodies to Xrn2 and are attempting to identify interacting protein partners to Xrn2. The generality of this termination mechanism is unknown; however, a further example of CoTC-mediated transcription termination has recently been described in the 3 '-flanking region of the mouse serum albumin gene (West et al. 2006a). Whereas in the human $\beta$-actin gene it appears that, due to the effect of transcriptional pausing (see below), the initial engagement of $5^{\prime} \rightarrow 3^{\prime}$ exonuclease with the transcript occurs at the free $5^{\prime}$ end generated by cleavage at the poly(A) site (Gromak et al. 2006).

\section{MOLECULAR TETHERING BY 3'-FLANKING REGION NONCODING RNA}

Apart from being a target of CoTC, 3 '-flanking region ncRNA appears to have a role in tethering of poly $(\mathrm{A})$-site sequences to pol II. We and other investigators have shown that endonucleolytic poly(A)-site cleavage of mammalian pre-mRNAs occurs some time after the poly(A)-site sequences have been transcribed (Baurén et al. 1998; Dye and Proudfoot 1999). This observation, coupled with evidence that the carboxy-terminal domain (CTD) of the large $\beta$-subunit of pol II has a role in $3^{\prime}$-end processing, leads us to a model in which a stable association of the poly(A) factors with the polymerase complex is established subse- 
quent to transcription of the poly(A)-site sequences. As pol II continues transcription, the interaction of the poly(A) signal with the polymerase complex would lead to the formation of an RNA loop composed of the 3'-flanking region transcript (Fig. 3D). This association appears to be maintained at least until transcript cleavage at the poly $(\mathrm{A})$ site takes place. Recent experiments in our laboratory and others have investigated the role of this structural loop in RNA 3'-end processing. It has been shown that cleavage of the nascent transcript, between the poly(A) site and termination sequences, leads to a reduction of poly(A)-site cleavage efficiency (Rigo et al. 2005). Thus, it appears that the ncRNA downstream from the poly(A) site acts as a molecular tether between the polymerase and the poly $(\mathrm{A})$ signal, somehow enhancing processing at the poly(A) site.

An interesting aspect of $3^{\prime}$-flanking region sequences is the presence of transcriptional pause elements that have been shown to be important in altering the efficiency of $3^{\prime}$ end processing and subsequent transcriptional termination (Yonaha and Proudfoot 2000; Gromak et al. 2006). It has previously been assumed that pause signals operated at the DNA level and involved the recruitment of DNA-binding proteins to a specific terminator/pause sequence as is the case for pol I in both humans and yeast (Paule and White 2000). However, evidence for a similar role of DNA-binding proteins in promoting pol II termination has not been forthcoming. We therefore cannot exclude the possibility that pol II transcriptional pausing involves ncRNA. Our current understanding of transcriptional pausing is that it might increase the dwell time of the polymerase complex at a particular position, downstream from the poly(A) site, and thereby enhance processing of the pre-mRNA. Alternatively, pause sequences might act at the RNA level, stabilizing the molecular tether between the poly(A) site and pol II, thus enhancing 3 '-end processing. This would in turn increase the likelihood of transcription termination.

A further potential role of ncRNA in pol II termination may relate to the occurrence of hybridization of premRNA with the gene DNA template forming so-called R loops. Significant R-loop formation has been shown to impair transcription elongation (Huertas and Aquilera 2003) and may have a role in intron splicing (Li and Manley 2005). Our laboratory is currently studying the presence of $\mathrm{R}$ loops in candidate 3'-flanking regions of several yeast genes. It is possible that R-loop formation between ncRNA and the DNA template could induce pausing of pol II as a way of promoting pol II termination.

\section{ROLES AND TURNOVER OF NONCODING RNA IN PRE-MRNA SPLICING}

Pre-mRNA splicing is the process by which introns, largely composed of ncRNA, are excised from the pre-mRNA transcript and adjacent exons are ligated together to form a continuous mRNA (Fig. 1B,C) (Moore et al. 1993). The importance of pre-mRNA splicing in gene expression cannot be exaggerated. In particular, it provides the molecular underpinning for the generation of different mRNA isoforms and consequently different proteins from between one-half to two-thirds of all human genes by alternative splicing (Modrek and Lee 2002). Furthermore, premRNA splicing has a pivotal role in the development of complex organisms. The evolution of splicing has recently been implicated as a driving force in the origin of the eukaryotic nucleus (Martin and Koonin 2006).

Although much research effort has gone into unraveling the details of splicing, there are still major gaps in our understanding of this complex process. One of these is the understanding of how splice site selection occurs. Splice site consensus sequences are remarkably simple. The splice donor (SD) site at the $5^{\prime}$ end of the intron is determined by a short sequence containing the conserved GU dinucleotide. The splice site at the $3^{\prime}$ end of the intron is more complex consisting of two sequences; the branch site that usually consists of a highly conserved A residue followed by a pyrimidine-rich tract and the splice acceptor (SA) site which is defined by a short sequence containing a conserved AG dinucleotide at the $3^{\prime}$ end (Proudfoot et al. 2002). Due to the length of introns and the fact that there are no obvious restraints on their internal sequence, introns often contain many of the relatively simple sequences that define splice signals. Occasionally, the arrangement of these so-called cryptic splice sites can define regions within the body of the intron as pseudoexons (Fig. 4A). However, splice site selection shows remarkable fidelity, using only the regular splice sites and generally ignoring cryptic splice sites and pseudoexons.

A critical advance in our understanding of splice site selection came with the exon definition hypothesis which suggested that identification of exons by recognition of their flanking splice sites marks them for retention in the mRNA and consequently marks the introns for removal by splicing (Berget 1995). Experimental evidence shows that exon definition is principally brought about by SR proteins bridging the exon and thus connecting splice factors binding to the flanking SA and SD splice sites (Fig. 4A). With the basic mechanism of SD-site selection understood, two further questions arise. First, how is the interaction between the chosen SD site maintained in the face of competition from cryptic SD sites within the intron? Second, especially in the case of transcription of very long introns, how is the correct SA site chosen from the many candidate SA sites that will be transcribed before it (Fig. 4A)? Recent data from ChIP analyses in yeast and mammalian cells show that splice factors are recruited to splice donor sites cotranscriptionally, possibly as soon as they emerge in the nascent transcript (Újvári and Luse 2004; Görnemann et al. 2005; Lacadie and Rosbash 2005). These data, combined with evidence for interaction of $5^{\prime}$ splice factors with elongating pol II and the extraordinary fidelity of SD-site selection (Maniatis and Reed 2002), give a strong indication that SDsite selection occurs on a "first come, first served" basis, through exon definition, and that a strong interaction between the SD site and the transcription complex is quickly formed. Presumably, it is this strong interaction that withstands competition from cryptic SD sites within the intron. Although SA-site selection in short introns may be based on simple sequence recognition, in long introns, this seems less likely. We suggest that the problem of splice site selection might be related to a speculative idea, concerning how pol II deals with the production of huge amounts of ncRNA when transcribing long introns (discussed below).

The classic two-step model of pre-mRNA splicing involves the formation of an intron lariat in step 1, which 
A

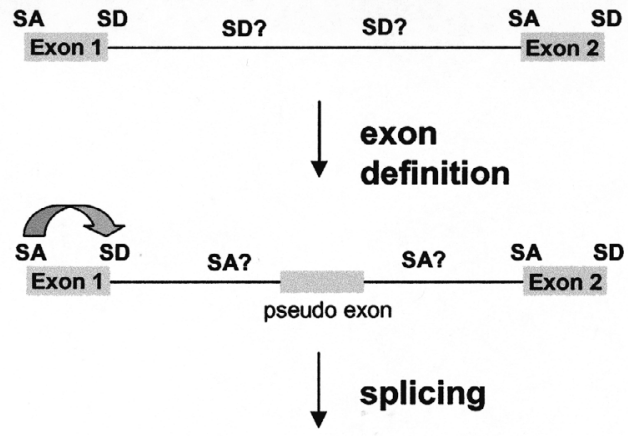

Exon 1 Exon 2

B

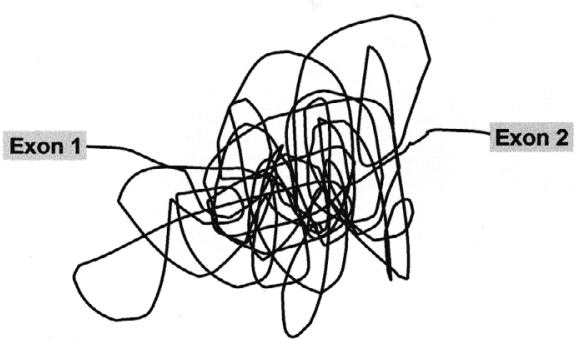

Figure 4. Turnover and roles of intronic ncRNA. $(A)$ Splice site selection and exon definition. Diagram showing position of splice sites in and around a hypothetical intron. SA and SD indicate splice acceptor site and splice donor site, respectively. SA? and SD? indicate cryptic splice sites. (Curved arrow) Interactions involved in exon definition. $(B)$ The problem of transcribing and processing long tracts of intron sequence. (Knotted line) Intronic pre-mRNA.

is released concomitant with exon ligation in step 2. This model is based on experiments carried out with nuclear extracts that are optimized for splicing in vitro and electron microscopic evidence for lariat-shaped splicing intermediates in vivo (Moore et al. 1993). Further evidence for this mechanism comes from analysis of unusual highly stable intron transcripts that are exported out of the nucleus (Clement et al. 1999, 2001). However, when one considers that transcription of some introns would result in the accumulation of vast amounts of pre-mRNA near the site of transcription, it appears unlikely that splicing can only occur as outlined above. Long tracts of unspliced pre-mRNA would occupy a lot of nuclear space, and the splicing machinery would encounter difficulty in finding the correct splice site within a mass of unspliced RNA (Fig. 4B). Studies in Drosophila have shown that splice sites located within certain long introns are used as "stepping stones," enabling the splicing out of the intron in segments (Hatton et al. 1998). Bioinformatic analysis shows that several genes in Drosophila might employ this recursive splicing mechanism to process long tracts of intronic ncRNA (Burnette et al. 2005).

A recent paper from our laboratory offers an additional explanation of how the transcription and splicing of long introns occur. Our study shows that cotranscriptional cleavage of a nascent intron using either the CoTC element from the $\beta$-globin gene 3 '-flanking region or a fast and efficient hammerhead ribozyme has no discernable effect on steady-state mRNA level (Fig. 5A) (Dye et al. 2006). The observation that a noncontinuous pre-mRNA can be efficiently spliced gives direct evidence for the cotranscriptional establishment of a strong interaction between the splice donor site at the $5^{\prime}$ end of the intron mentioned above. We suggest that the establishment of this strong interaction, coupled with continued transcription, results in the formation of an RNA loop similar to that which occurs downstream from the poly(A) site (Fig. $5 \mathrm{~B}$ ). We further suggest that the loop is cotranscriptionally cleaved, allowing access of the RNA degradation machin-

A
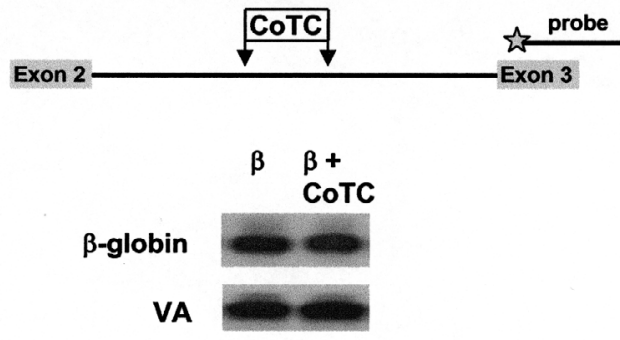

B
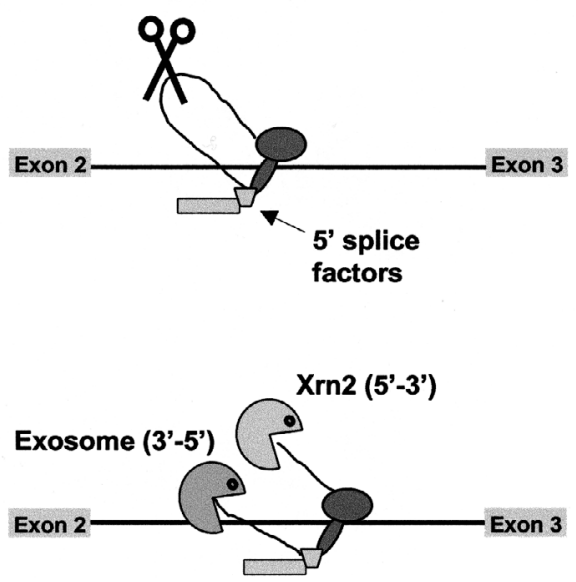

Figure 5. Cotranscriptional intron ncRNA degradation. $(A)$ Cotranscriptional cleavage of intron transcripts does not influence splicing. (Upper panel) Diagram of intron 2 and flanking exons 2 and 3 of the $\beta$ and $\beta+$ CoTC globin gene constructs; (lower panel) nuclease $\mathrm{S} 1$ analysis of $\beta$ and $\beta+\mathrm{CoTC}$ transcripts. The end-labeled probe used in this experiment is shown in the diagram in the upper panel. The VA signal derives from the cotransfected VA plasmid and controls for transfection efficiency and RNA recovery. (B) (Upper panel) Tethering of premRNA to pol II. In the diagram (not to scale): (solid line) $\beta$-globin intron-2 DNA template; (single curved line) nascent transcript; (gray box with black outline) tethered exon; (rhombus) splice factors associated with the intron-2 splice donor site; (scissors) cotranscriptional cleavage of the nascent intron transcript. (Lower panel) Cotranscriptional intron degradation. The "Pac Man" symbols represent 3'-5' and 5'-3' exonucleases. 
ery to remove ncRNA parts of the pre-mRNA as they are synthesized (Fig. 5B). This "exon tethering" model suggests that some introns do not act as connectors between splice sites at the RNA level and that the lariat structure may not be formed, as envisaged in the standard premRNA splicing model. Having proven that pre-mRNA splicing can occur across a noncontinuous intron, we hypothesize that target sequences for an endonucleolytic cotranscriptional cleavage activity, such as that found in the 3 -flanking region of the $\beta$-globin gene, might reside in endogenous mammalian introns. Consequently, we are now searching for examples of cotranscriptional cleavage of intronic ncRNA in endogenous genes.

CoTC generates free $5^{\prime}$ and $3^{\prime}$ ends that are substrates of the nuclear RNA degradation machinery. We suggest that cotranscriptional degradation of intron sequences could resolve some of the problems pol II must encounter when producing very long tracts of ncRNA. Rapid intron degradation could eliminate cryptic splice sites from the intron transcript before they have a chance to compete for splicing. This hypothesis invokes another mechanism of regulating splicing. Either ncRNA sequences to be degraded are somehow marked for degradation or sequences to be protected are marked for protection. An important objection to this model would be that unrestrained RNA degradation, initiating within the intron transcript, could lead to the destruction of the entire premRNA. However, our observation that splicing is completely unaffected by CoTC indicates that this is not the case. We suggest that the important splice signals, located at the $5^{\prime}$ and $3^{\prime}$ ends of the cotranscriptionally cleaved intron transcript, are protected from degradation, This protection could be mediated by interaction between the pre-mRNA with the transcription complex, splice factors, and hnRNPs. Binding of these factors to the transcript might hinder or slow down RNA exonuclease activity.

Introns are not simply featureless ncRNA linkers between exons, they also contain information. The importance of intronic splice signals, generally located at the $5^{\prime}$ and $3^{\prime}$ exon/intron junctions, in the regulation of gene expression is well established. There is also evidence for roles of other intronic sequences. Some introns contain sequences that encode small regulatory RNAs, including miRNAs and snoRNAs (Mattick and Makunin 2006) as well as important regulatory signals such as splicing enhancers and silencers (McCullough and Berget 2000; Sun and Chasin 2000; Wagner et al. 2005). Clearly, these sequences must be protected from degradation. In this regard, it is interesting to note that some miRNAs appear to be cotranscriptionally excised from their intronic RNA background (V.N. Kim, pers. comm.). It is exciting to speculate on the possibility that cotranscriptional excision of regulatory RNAs from introns could have been a driving force in the evolution of exon tethering.

In summary, our studies of 3 '-flanking region and intron transcripts have shown that these intragenic ncRNAs have some very important properties. Intragenic ncRNA can act as a molecular tether connecting transcribed RNA-processing signals to elongating polymerase and thus enhancing pre-mRNA processing. They can also act as targets for cotranscriptional cleavage, which is instrumental in the process of RNA pol II tran- scriptional termination on some gene templates. Intronic ncRNA also forms loops that may also be targets for CoTC. CoTC and subsequent transcript degradation introduces another level of complexity to the already complex process of pre-mRNA splicing. However, cotranscriptional intron degradation might also solve the dynamic and spatial problems faced by the cell in transcribing long tracts of noncoding intron sequence and might also prevent competition by cryptic splice sites.

\section{TURNOVER AND FUNCTION OF INTERGENIC NCRNA TRANSCRIPTS}

It has become apparent in recent years that transcription of ncRNA by pol II is not only confined within the boundaries of genes. Transcriptional analysis of a variety of eukaryotic genomes has revealed high levels of widespread intergenic transcription (Bertone et al. 2004; Stolc et al. 2005; Samanta et al. 2006). In general, this intergenic ncRNA is highly unstable. In the yeast $S$. cerevisiae, it has been shown that these cryptic unstable transcripts (CUTs) are stabilized when the major RNA degradation apparatus, the multisubunit exosome, is inactivated by gene knockout (Wyers et al. 2005), implicating an important role for this complex in ncRNA turnover. CUTs are of unknown function; however, it is thought that some may be involved in gene regulation processes such as that demonstrated for the SRG1 gene transcript that regulates $S E R 3$ gene expression by selective transcriptional interference (Martens et al. 2006).

In the yeast Schizosaccharomyces pombe, transcription of intergenic ncRNA is associated with epigenetic regulation of centromeric sequences (Volpe et al. 2002). It has been shown that intergenic transcripts are involved in maintaining a repressed chromatin state through RNAi pathways that cotranscriptionally recruit chromatin remodeling activities (Cam and Grewal 2004; Buhler et al. 2006). Interestingly, these transcripts are synthesized not only by pol II, but also by an RNA-dependent RNA polymerase (Rdp) activity. Similarly, in plants, intergenic transcripts synthesized by an Rdp activity and a dedicated RNA polymerase, termed pol IV, act to set up appropriate chromatin structures throughout the plant genome in response to developmental and viral cues (Herr et al. 2005). The possibility that such chromatin-associated intergenic transcription is prevalent in all eukaryotic genomes remains to be established. However, work in our laboratory, which we describe below, has shown widespread intergenic transcription across the human $\beta$-globin gene cluster that may have a similar regulatory role in the establishment of chromatin structure.

We originally detected transcription of intergenic regions in the human $\beta$-globin gene cluster, shown in the diagram in Figure 6, of erythroid and non-erythroid cells by nuclear run-on analysis following various induction treatments (Ashe et al. 1997; Plant et al. 2001). We also determined that transcription of this ncRNA likely initiates in the repetitive ERV-9 LTR (long terminal repeat) region sequence (Plant et al. 2001). These intergenic transcripts proved to be difficult to analyze due to their intrinsic instability and rapid turnover. However, in a later study, it was found that treatment of non-erythroid cells 
with the histone deacetylase inhibitor trichostatin A (TSA) caused widespread activation of intergenic transcription across the $\beta$-globin gene cluster which was detectable using high-amplification reverse transcriptase (RT)-PCR analysis. Figure 6 shows RT-PCR analysis of transcripts located downstream from the ERV-9 sequence. Similar results were obtained throughout the gene cluster (Haussecker and Proudfoot 2005). Interestingly, both sense and antisense transcripts were detected and, remarkably, RNAi-mediated knockdown of Dicer resulted in a considerable increase in transcript levels, suggesting that these intergenic transcripts are substrates for RNAi-related turnover, similar to that originally defined in $S$. pombe centromeres (Cam and Grewall 2004). Interestingly, genic as well as intergenic transcripts were induced by TSA treatment of HeLa cells. These genic transcripts were probably generated by transcriptional readthrough from intergenic regions. Strikingly, only the unspliced transcripts of each of the globin genes were susceptible to Dicer as shown by the relative elevation in their abundance, as compared to that of the spliced transcripts, following Dicer knockdown (Fig. 6) (Haussecker and Proudfoot 2005). Taken together, these data indicate that aberrant transcripts, whether of intergenic or unprocessed genic origin, are substrates for an RNAi-mediated degradation pathway from which, presumably, induced spliced transcripts as well as authentic genic transcripts are somehow sequestered. A connection of these results with chromatin silencing was revealed when the $\beta$-globin locus was tested for changes in chromatin structure following Dicer knockdown. As shown in Figure 7, Dicer knockdown results in an increase in histone acetylation across the cluster, suggesting that Dicer-mediated degradation of the $\beta$-globin locus intergenic transcripts may well relate to normal repression of this cluster in non-erythroid cells.

\section{CONCLUSIONS}

We have described studies of noncoding pol II transcripts from both within and outside the boundaries of the gene. Our account of intragenic ncRNA transcripts, derived from the introns and 3 '-flanking regions of proteinencoding genes, indicates that they have important roles both in connecting RNA processing signals to transcribing pol II and in serving as substrates for RNA degradation. Although a high energetic cost must be associated with the transcription and turnover of this apparently nonproductive ncRNA, it appears from our studies that the actual degradation process may have evolved in concert with the splicing, 3'-end processing, and transcriptional termination mechanisms and have an intrinsic role in them. Our studies of intergenic ncRNA transcription indicate that RNAirelated mechanisms are involved in regulating intergenic transcription in the human $\beta$-globin gene cluster and further suggest that RNAi-dependent chromatin silencing in vertebrates is not restricted to the centromeres. Furthermore, we speculate that the intergenic transcripts in the $\beta$-globin cluster might be representative of widespread intergenic ncRNA throughout the human genome and, by extrapolation, all higher eukaryotes.

Previous work from our laboratory (O'Sullivan et al. 2004) has demonstrated that transcription by pol II is associated with the formation of a gene-loop structure in which the promoter and terminator regions of the gene move into close physical contact following gene activa-
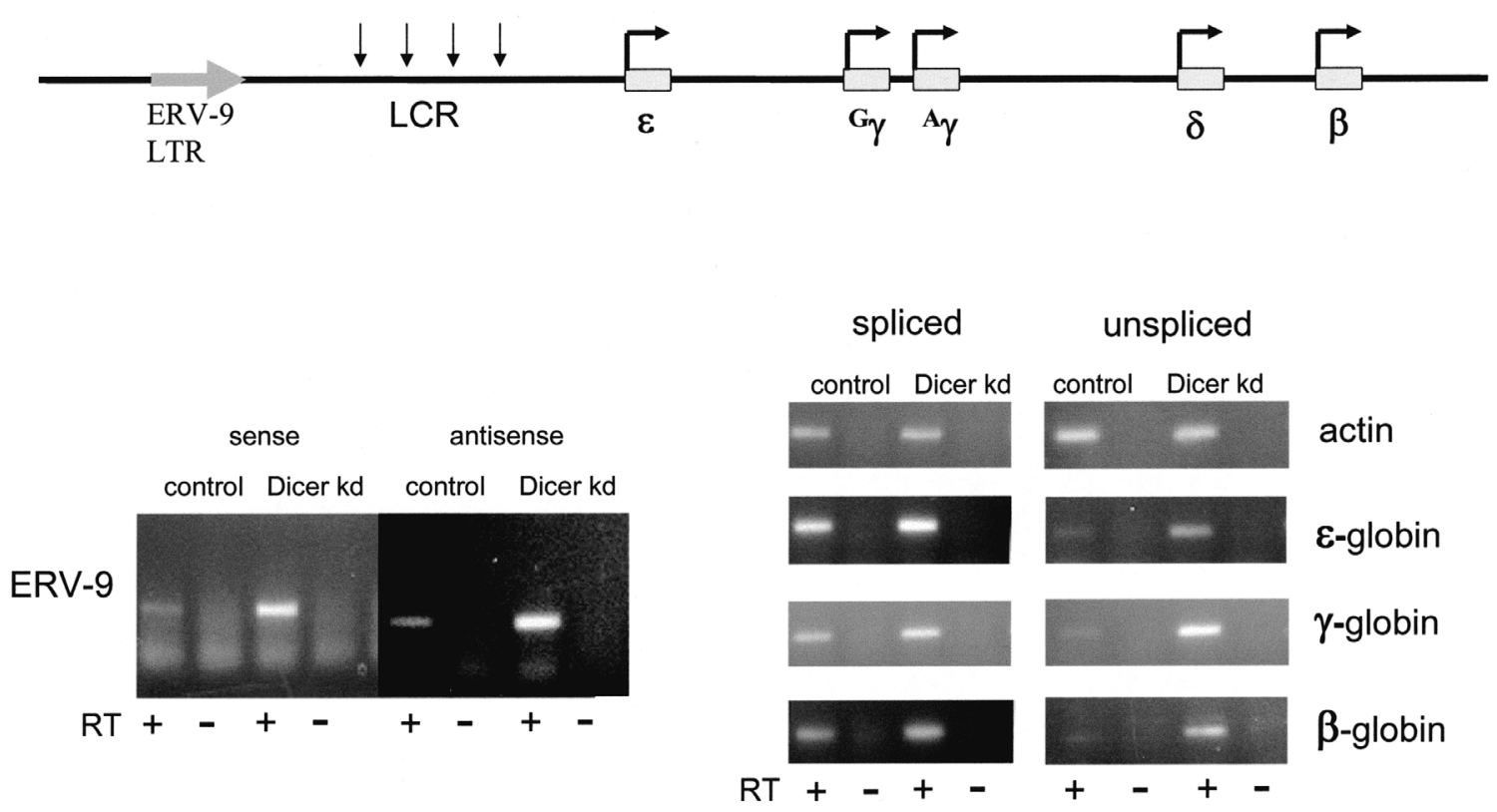

Figure 6. Intergenic transcription in the human $\beta$-globin gene cluster in TSA-treated HeLa cells. Diagram of the human $\beta$-globin gene cluster showing positions of five structural genes as well as the locus control region and ERV-9 LTR. Below the locus map, selective RT-PCR analysis is shown for the ERV-9 region as well as genic regions using primers specific for both spliced and unspliced transcripts. Nuclear RNA was isolated from TSA-induced HeLa cells with or without Dicer siRNA treatment to knockdown Dicer expression. All RT-PCRs were controlled by omitting reverse transcriptase to rule out DNA contamination (-RT). Equal amounts of nuclear RNA were used in each separate experimental panel. For ERV-9 antisense, RT-PCR was carried out using a higher number of cycles. 


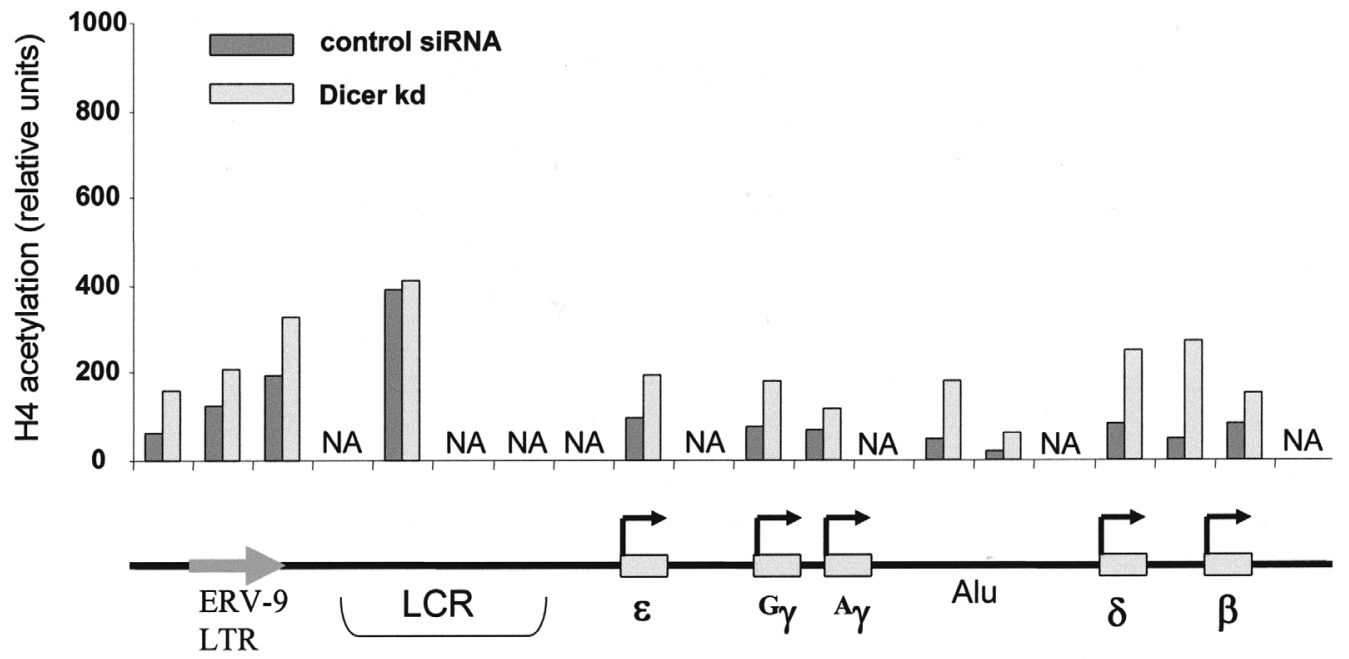

Figure 7. Changes in chromatin structure of the human $\beta$-globin gene cluster following Dicer knockdown. ChIP analysis of histone $\mathrm{H} 4$ acetylation across the human $\beta$-globin gene cluster in control or Dicer siRNA-treated HeLa cells. NA denotes not assayed. Positions of ChIP signals are aligned to the $\beta$-globin locus map below. For further details, see Haussecker and Proudfoot (2005).

tion. These observations have been confirmed and extended in a study showing that specific promoter and terminator factors are required for loop formation (Ansari and Hampsey 2005). Consequently, it is apparent that genes actively transcribed by pol II may possess two levels of loop conformation: gene loops and intergenic ncRNA loops (Fig. 8). This RNA and DNA loop arrangement suggests a high level of structural organization for actively expressed genes We propose that intragenic ncRNA transcription may occur within this type of geneloop structure. In contrast, intergenic ncRNA transcription may occur outside of such a loop structure and

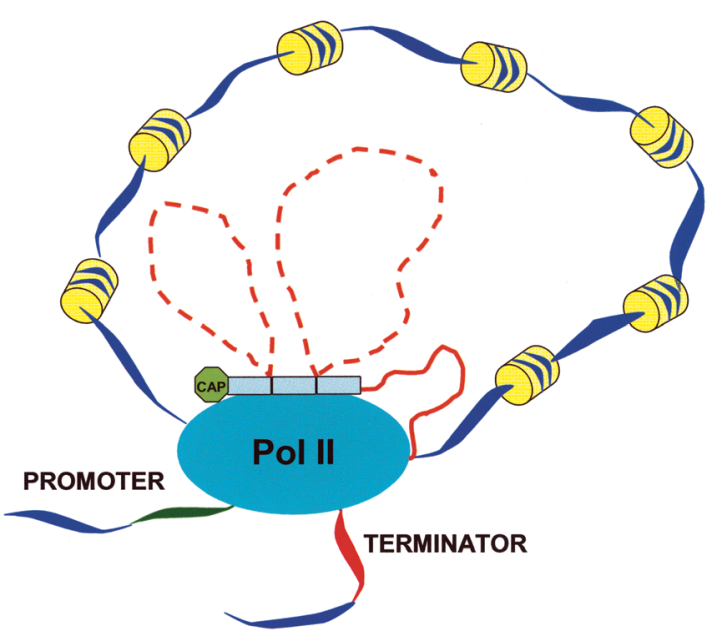

Figure 8. Gene loops and ncRNA loops may combine to define productive transcription units. Model showing the tight structural constraints that may exist to define a gene that is expressing pre-mRNA. Intergenic transcription that relates to RNAi effects may be excluded from such gene-loop regions. Diagram shows DNA associated with nucleosomes (cylinders) held at the promoter and terminator regions by the pol II complex. Tethered exons bind to the pol II complex with introns (dashed red lines) and 3 '-flanking region (solid red line) looped out. therefore intergenic transcription can have a role in chromatin RNAi effects, rather than resulting in productive mRNA synthesis. This hypothesis requires direct testing and is the subject of ongoing research in our laboratory.

\section{ACKNOWLEDGMENTS}

We thank members of the Proudfoot lab for helpful discussion and comments. This work was supported by a Programme Grant from the Wellcome Trust to N.J.P. D.H. was supported by a postgraduate scholarship from the Darwin Trust. S.W. was supported by an EPA research studentship.

\section{REFERENCES}

Ansari A. and Hampsey M. 2005. A role for the CPF 3'-end processing machinery in RNAP II-dependent gene looping. Genes Dev. 19: 2969.

Ashe H.L., Monks J., Wijgerde M., Fraser P., and Proudfoot N.J. 1997. Intergenic transcription and transinduction of the human $\beta$-globin locus. Genes Dev. 11: 2494.

Bartel D.P. 2004. MicroRNAs: Genomics, biogenesis, mechanism and function. Cell 116: 281.

Bartlett J., Blagojevic J., Carter D., Eskew C., Fromaget M., Job C., Shamser M., Trinidade I.F., Xu M., and Cook P.R. 2006. Specialised transcription factories. Biochem. Soc. Symp. 73: 67.

Baurén G., Belikov S., and Wieslander L. 1998. Transcriptional termination in the Balbiana ring 1 gene is closely coupled to 3 '-end formation and excision of the $3^{\prime}$ terminal intron. Genes Dev. 12: 2759.

Berget S.M. 1995. Exon recognition in vertebrate splicing. $J$. Biol. Chem. 270: 2411.

Bertone P., Stolc V., Royce T.E., Rozowsky J.S., Urban A.E., Zhu X., Rinn J.L., Tongprasit W., Samanta M., Weismann S., et al. 2004. Global identification of human transcribed sequences with genome tiling arrays. Science 306: 2242.

Birse C.E., Minvielle-Sebastia L., Lee B.A., Keller W., and Proudfoot N.J. 1998. Coupling termination of transcription to messenger RNA maturation in yeast. Science 280: 298.

Buhler M., Verdel A., and Moazed D. 2006. Tethering RITS to a nascent transcript initiates RNAi- and heterochromatindependent gene silencing. Cell 125: 873. 
Burnette J.M., Miyamoto-Sato E., Schaub M.A., Conklin J., and Lopez A.J. 2005. Subdivision of large introns in Drosophila by recursive splicing at nonexonic elements. Genetics 170: 661.

Cam H. and Grewal S.I. 2004. RNA interference and epigenetic control of heterochromatin assembly in fission yeast. Cold Spring Harbor Symp. Quant. Biol. 69: 419.

Clement J.Q., Maiti S., and Wilkinson M.F. 2001. Localisation and stability of introns spliced from the Pem homeobox gene. J. Biol. Chem. 276: 16919.

Clement J.Q., Qian L., Kaplinsky N., and Wilkinson M.F. 1999. The stability and fate of a spliced intron from vertebrate cells. RNA 5: 206.

Connelly S. and Manley J.L. 1988. A functional mRNA polyadenylation signal is required for transcription termination by RNA polymerase II. Genes Dev. 2: 440.

Dye M.J. and Proudfoot N.J. 1999. Terminal exon definition occurs cotranscriptionally and promotes termination of RNA polymerase II. Mol. Cell 3: 371 .

2001. Multiple transcript cleavage precedes polymerase release in termination by RNA polymerase II. Cell 105: 669 .

Dye M.J., Gromak N., and Proudfoot N.J. 2006. Exon tethering in transcription by RNA polymerase II. Mol. Cell 21: 849 .

Görnemann J., Kotovic K.M., Hujer K., and Neugebauer K.M. 2005. Cotranscriptional spliceosome assembly occurs in a stepwise fashion and requires the cap binding complex. Mol. Cell 19: 53.

Gromak N., West S., and Proudfoot N.J. 2006. Pause sites promote transcriptional termination of mammalian RNA polymerase II. Mol. Cell. Biol. 26: 3986.

Hatton A.R., Subramaniam V., and Lopez A.J. 1998. Generation of alternative ultrabithorax isoforms and stepwise removal of a large intron by resplicing at exon-exon junctions. Mol. Cell 2: 787 .

Haussecker D. and Proudfoot N.J. 2005. Dicer-dependent turnover of intergenic transcripts from the human beta globin gene cluster. Mol. Cell. Biol. 25: 9724.

Herr A.J., Jensen M.B., Dalmay T., and Baulcombe D.C. 2005. RNA polymerase IV directs silencing of endogenous DNA. Science 308: 118.

Huertas P. and Aguilera A. 2003. Cotranscriptionally formed DNA:RNA hybrids mediate transcription elongation impairment and transcription-associated recombination. Mol. Cell 12: 711 .

Kim M., Krogan N.J., Vasiljeva L., Rando O.J., Nedea E., Greenblatt J.F., and Buratowski S. 2004. The yeast Rat1 exonuclease promotes transcription termination by RNA polymerase II. Nature 432: 517.

Lacadie S.A. and Rosbash M. 2005. Cotranscriptional spliceosome assembly dynamics and the role of U1 snRNA:5'ss base pairing in yeast. Mol. Cell 19: 65 .

Lewis B.P., Burge C.B., and Bartel D.P. 2005. Conserved seed pairing, often flanked by adenosines, indicates that thousands of human genes are microRNA targets. Cell 120: 15 .

Li X. and Manley J.L. 2005. Inactivation of the SR protein splicing factor ASF/SF2 results in genomic instability. Cell 122: 365 .

Maniatis T. and Reed R. 2002. An extensive network of coupling among gene expression machines. Nature 416: 499.

Maniatis T. and Tasic B. 2002. Alternative pre-mRNA splicing and proteome expansion in metazoans. Nature 418: 236.

Martens J.A., Wu P.-Y., and Winston F. 2006. Regulation of an intergenic transcript controls adjacent gene transcription in Saccharomyces cerevisiae. Genes Dev. 19: 2695.

Martin W. and Kroonin E.V. 2006. Introns and the origin of nucleus-cytosol compartmentalisation. Nature 440: 41.

Mattick J.S. and Makunin I.V. 2006. Non-coding RNA. Hum. Mol. Genet. 15: 17.

McCullough A.J. and Berget S.M. 2000. An intronic splicing enhancer binds U1 snRNPs to enhance splicing and select $5^{\prime}$ splice sites. Mol. Cell. Biol. 20: 9225.
Modrek B. and Lee C. 2002. A genomic view of alternative splicing. Nat. Genet. 30: 13.

Moore M.J., Query C.C., and Sharp P.A. 1993. Splicing of precursors to mRNA by the spliceosome. In The RNA world (ed. R.F. Gesteland and J.F. Atkins), p. 303. Cold Spring Harbor Laboratory Press, Cold Spring Harbor, New York.

Moore M.J. 2005. From birth to death: The complex lives of eukaryotic mRNAs. Science 309: 1514.

O'Sullivan J.M., Tan-Wong S.M., Morillon A., Lee B., Coles J., Mellro J., and Proudfoot N. 2004. Gene loops juxtapose promoters and terminators in yeast. Nat. Genet. 36: 1014.

Paule M.R. and White R.J. 2000. Transcription by RNA polymerases I and III. Nucleic Acids Res. 28: 1283.

Plant K.E., Routledge S.J.E., and Proudfoot N.J. 2001. Intergenic transcription in the human $\beta$-globin gene cluster. Mol. Cell. Biol. 21: 6507.

Proudfoot N.J., Furger A., and Dye M.J. 2002. Integrating mRNA processing with transcription. Cell 108: 501.

Rigo F., Kazerouninia A., Nag A., and Martinson H. 2005. The RNA tether from the poly(A) signal to the polymerase mediates coupling of transcription to cleavage and polyadenylation. Mol. Cell 20: 733.

Samanta M.P., Tongprasit W., Sethi H., Chin C.-S., and Stolc V. 2006. Global identification of noncoding RNAs in Saccharomyces cerevisiae by modulating an essential RNA processing pathway. Proc. Natl. Acad. Sci. 103: 4192.

Strachan T. and Read A.P. 1999. Organisation and distribution of human genes, chapter 7.2. In Human molecular genetics, 2nd edition. Garland Science, New York.

Stolc V., Samanta M.P., Tongprasit W., Sethi H., Liang S., Nelson D.C., Hegeman A., Nelson C., Rancour D., Bednarek S., et al. 2005. Identification of transcribed sequences in Arabidopsis thaliana by using high resolution genome tiling arrays. Proc. Natl. Acad. Sci. 102: 4453.

Sun H. and Chasin L. 2000. Multiple splicing defects in an intronic false exon. Mol. Cell. Biol. 20: 6414.

Tantravahi J., Alvira M., and Falck-Pedersen E. 1993.

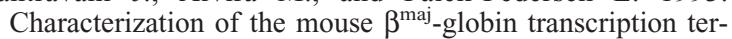
mination region: A spacing sequence is required between the poly(A) signal sequence and multiple downstream termination elements. Mol. Cel. Biol. 13: 578.

Újvári A. and Luse L.S. 2004. Newly initiated RNA encounters a factor involved in splicing immediately upon emerging from within RNA polymerase II. J. Biol. Chem. 279: 49773.

Volpe T.A., Kidner C., Hall I.M., Teng G., Grewal S.I., and Martienssen R.A. 2002. Regulation of heterochromatic silencing and histone H3 lysine-9 methylation by RNAi. Science 297: 1818.

Wagner E.J., Baraniak A.P., Sessions O.M., Mauger D., Moskowitz E., and Garcia-Blanco M.A. 2005. Characterization of the intronic splicing silencers flanking FGFR2 exon IIIb. J. Biol. Chem. 280: 14017.

West S., Gromak N., and Proudfoot N.J. 2004. Human 5' $\rightarrow 3^{\prime}$ exonuclease Xrn2 promotes transcription termination at cotranscriptional cleavage sites. Nature 432: 522.

West S., Zaret K., and Proudfoot N.J. 2006a. Transcriptional termination sequences in the mouse serum albumin gene. $R N A$ 12: 655 .

West S., Gromak N., Norbury C.J., and Proudfoot N.J. 2006 b. Adenylation and exosome-mediated degradation of cotranscriptionally cleaved pre-messenger RNA in human cells. Mol. Cell 21: 437.

Whitelaw E. and Proudfoot N. 1986. $\alpha$-Thalassaemia caused by a poly(A) site mutation reveals that transcriptional termination is linked to $3^{\prime}$ end processing in the human $\alpha 2$ globin gene. EMBO J. 5: 2915.

Wyers F., Rougemaile M., Badis G., Rouselle J.-C., Dufour M.E., Boulay J., Régnault B., Devaux F., Namane A., Séraphin B., et al. 2005. Cryptic Pol II transcripts are degraded by a nuclear quality control pathway involving a new poly(A) polymerase. Cell 121: 725 .

Yonaha M. and Proudfoot N.J. 2000. Transcriptional termination and coupled polyadenylation in vitro. EMBOJ. 19: 3770 . 


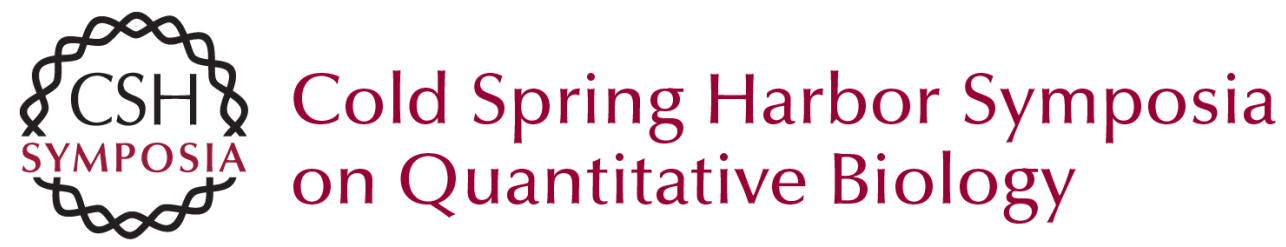

\section{Turnover and Function of Noncoding RNA Polymerase II Transcripts}

M.J. DYE, N. GROMAK, D. HAUSSECKER, et al.

Cold Spring Harb Symp Quant Biol 2006 71: 275-284

Access the most recent version at doi:10.1101/sqb.2006.71.040

References This article cites 53 articles, 27 of which can be accessed free at: http://symposium.cshlp.org/content/71/275.full.html\#ref-list-1

License

Email Alerting Receive free email alerts when new articles cite this article - sign up in Service the box at the top right corner of the article or click here. 